\title{
On the boundedness of running-in attractors based on recurrence plot and recurrence qualification analysis
}

\author{
Guodong SUN, Hua ZHU*, Cong DING, Yu JIANG, Chunling WEI \\ School of Mechatronic Engineering, China University of Mining and Technology, Xuzhou 221116, China \\ Received: 11 July 2017 / Revised: 06 December 2017 / Accepted: 23 April 2018 \\ (C) The author(s) 2018. This article is published with open access at Springerlink.com
}

\begin{abstract}
A feature parameter was proposed to quantitatively explore the boundedness of running-in attractors; its variation throughout the friction process was also investigated. The enclosing radius $R$ was built with recurrence plots (RPs) and recurrence qualification analysis (RQA) by using the time delay embedding and phase space reconstruction. Additionally, the typology of RPs and the recurrence rate (RR) were investigated to verify the applicability of $R$ in characterizing the friction process. Results showed that $R$ is larger at the beginning, but exhibits a downward trend in the running-in friction process; $R$ becomes smooth and trends to small steady values during the steady-state friction period, and finally shows an upward trend until failure occurs. The evolution of $R$, which corresponded with the typology of RPs and RR during friction process, can be used to quantitatively analyze the variation of the running-in attractors and friction state identifacation. Hence, $R$ is a valid parameter, and the boundedness of running-in attractors can offer a new way for monitoring the friction state of tribological pairs.
\end{abstract}

Keywords: running-in attractor; boundedness; enclosing radius; recurrence plot; dynamic evolvement

\section{Introduction}

The friction coefficient (or "coefficient of friction", $\mathrm{COF}$ ) generated from friction couples is an important information carrier of friction conditions, directly reflecting wear state and wear mode [1,2]. Owing to their strong dependence on a tribological system and their time varying nature, friction signals inevitably display nonlinear and complex characteristics [3]. The study of the COF based on nonlinear theory has gained much attention over the years, particularly with respect to the field of fractal and chaos [4].

The sequence signals extracted from friction systems, including friction force, friction coefficient, frictioninduced vibration, and friction temperature, have been proven to be chaotic, and the output phase spaces show obvious low dimension $[5,6]$. The phase trajectory, obtained by connecting the phase points in turn, was demonstrated to intuitively describe the time evolution process of a system $[7,8]$. The attractors are spontaneous ordered spatiotemporal structures of phase trajectory during the running-in process; thus, the attractors in tribo-system are also referred to as running-in attractors. As typical chaotic attractors, the running-in attractors have the properties of boundedness, fractal-dimensionality, and intrinsic randomness [9], which can be characterized by fractal and chaotic parameters, including fractal and multifractal parameters [10,11], Lyapunov exponent [12], entropy [13], and predictability [14]. Each measurement presents and emphasizes the attractors from a different perspective. It has been widely confirmed that both the fractal dimension and multifractal spectra play a vital role in characterizing the fractal structures, while the Lyapunov exponent contributes to measuring the speed of divergence or convergence of nearby orbits in phase space, and the entropy measures the complexity of the attractors. Additionally,

* Corresponding author: Hua ZHU, E-mail: zhuhua83591917@163.com 
Zhou et al. [15] had successfully introduced predictability as a quantitative statistical judgment of the intrinsic randomness degree in running-in attractors.

The study of running-in attractors focuses not only on their properties and characterization parameters, but also on their extensive use in wear condition identification. Zhu et al. [16] discovered that running-in attractors are shaped during the running-in process. Sun et al. [17] stated that the evolvement of the friction vibration attractors corresponds to changes in wear state from the running-in stage to the stable wear stage. Zhou et al. [18] indicated that the evolution of friction temperature attractors abided by the law of "forming-keeping-disappearing", which was consistent with the stages of "running-in, steadystate, and increasing friction". Liu et al. [19] analyzed the cross correlation between tangential and normal friction vibration, and suggested that the chaotic attractors of both signals can be used to describe variations in the running-in process. Jiang et al. [20] developed a reliable method for fault diagnosis for gear boxes based on the chaotic degree of frictioninduced vibration attractors; the results showed that the chaotic attractor was maintained in a steady state, and the chaotic degree was related to fault severity.

The above-mentioned information and references are only a fraction of existing research papers that discuss running-in attractors, and there remains some unresolved issues. Firstly, as noted in Ref. [16, 18-20], the friction states can be recognized by the evolution of the phase trajectory of running-in attractors. However, there is no quantitative description of the degree of convergence of the running-in attractors, thus the existing studies on wear condition identification by phase space trajectory merely rest on qualitative analysis [21, 22]. Additionally, effective characterization parameters to appraise the boundedness of the running-in attractors are lacking. Zhang et al. [23] indicated that the bounds of a domain containing all compact invariant sets obtained in many cases can be used not only for theoretical studies of chaotic attractors, but also for estimating for the Haudorff dimension and/or for further numerical researches. Furthermore, it has been ascertained that there are obvious shortcomings in the characterization of rather short-time nonstationary sequences with the method of entropy, fractal dimension, and the maximum Lyapunov exponent [24, 25].

The primary goal for this paper is to put forward a feature parameter to quantitatively characterize the boundedness of running-in attractors, and to further investigate the evolvement of the nonlinear dynamic behavior of tribological systems by describing the variation in the proposed parameter. Moreover, recurrence plots (RPs) and recurrence quantification analysis (RQA) are explored to verify the proposed parameter. This paper is organized as follows. In Section 2, the friction experiments are described, and the COF signals are acquired throughout the friction process. In Section 3, measurement of the enclosing radius $R$ based on RPs and RQA is introduced to define the boundedness of running-in attractors. In Section 4, the morphological evolution of RPs, and the evolution of feature parameters are further studied. Finally, the major conclusions of the paper are summarized in Section 5.

\section{Experiments}

\subsection{Apparatus}

A self-regulating rotational motion tribometer illustrated in Fig. 1(a) was applied to conduct friction experiments. It can be clearly seen that the upper ring sample (Fig. 1(b)) was fixed into the ring holder by a locking bolt and locating pin, while a center hole and a mill groove were machined on the disc holder to locate the lower disc sample (Fig. 1(c)). Moreover, the wedge-ended pin was used to ensure relative stability of the disc sample. Rotary motion of the main shaft, driven by a motor, was carried through the ring holder to the upper sample, causing relative sliding between the contact surfaces. A frequency converter was applied to realize rotational speed adjustment, and the normal load was adjusted by weight. The COF was then calculated via the collected friction torque signal, which was measured using the static torque sensor mounted on the disc holder bottom, and then uploaded to the computer via the signal collecting device. With $\mu$ as the COF and $M$ as friction torque, the relationship is given via the following formula [26]: 


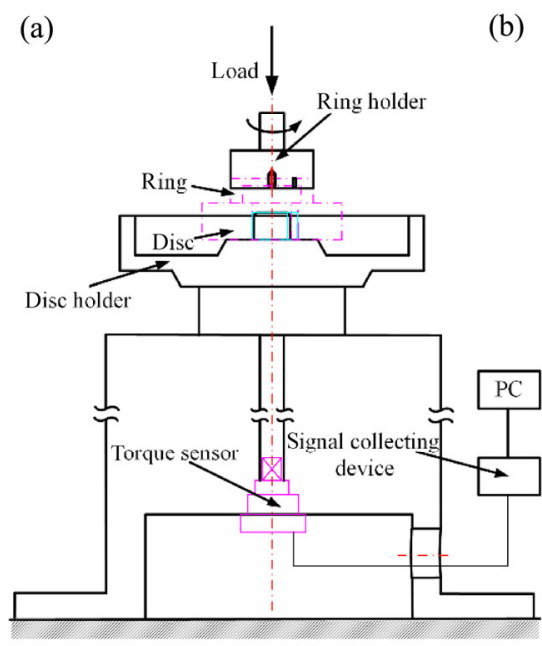

Schematic of rotating tribometer (b)
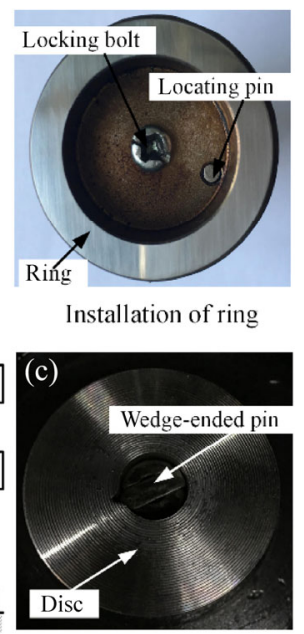

Installation of disc

Fig. 1 Schematic of rotating tribometer and installation of specimen.

$$
\mu=\frac{3\left(R_{1}+R_{2}\right)}{2 P\left(R_{1}^{2}+R_{1} R_{2}+R_{2}^{2}\right)} M
$$

where $R_{1}$ and $R_{2}$ are the outer and inner radius of the ring specimen, respectively. $P$ is the normal load applied on the contact surface.

\subsection{Test samples and test conditions}

The lower disc sample was made up of AISI 1045 steel with an HB194 hardness and was cut into $\Phi 46 \times 12 \mathrm{~mm}$ segments. The upper ring sample was made of AISI 52100 steel that was hardened up to $698 \mathrm{HB}$. The ring sample had an outer diameter of $34 \mathrm{~mm}$ and an inner diameter of $24 \mathrm{~mm}$. Thus, the nominal contact area was $455.53 \mathrm{~mm}^{2}$, and the equivalent radius was $14.5 \mathrm{~mm}$. The initial surface roughness of the ring and disc were $0.036-0.040 \mu \mathrm{m}$ and $5.60-5.90 \mu \mathrm{m}$, respectively. It is noted that both rings and discs were carefully cleaned with anhydrous ethanol via ultrasonic bath prior to being used in the friction experiments.

Four tests were carried out under different normal loads and velocities, and the experimental conditions were summarized in Table 1 . The samples were lubricated with CD 15W-40 type machine oil with a viscosity of $12.5-16.3$ at $100{ }^{\circ} \mathrm{C}$, with the flash point temperature higher than $215^{\circ} \mathrm{C}$ and freezing point temperature lower than $-20{ }^{\circ} \mathrm{C}$. Prior to tests, a volume of $0.2 \mathrm{~mL}$ lubricating oil was added to the contact surfaces and no more oil was supplied during

Table 1 Experimental conditions parameters in rotating friction tests.

\begin{tabular}{cccc}
\hline Test run & $\begin{array}{c}\text { Velocity } \\
(\mathrm{m} / \mathrm{s})\end{array}$ & $\begin{array}{c}\text { Normal load } \\
(\mathrm{N})\end{array}$ & $\begin{array}{c}\text { Normal pressure } \\
(\mathrm{MPa})\end{array}$ \\
\hline 1 & 0.76 & 480 & 1.05 \\
2 & 1.06 & 480 & 1.05 \\
3 & 0.76 & 580 & 1.27 \\
4 & 1.06 & 580 & 1.27 \\
\hline
\end{tabular}

the tests. Furthermore, the tests were carried out at room temperature. The COF signal was measured with a sampling frequency of $10 \mathrm{~Hz}$.

\subsection{Test results}

Any processed signal inevitably contains an abundance of noise, which can not only degrade the accuracy of parameters as-calculated, but also obscure or even destroy the inherent principles of the signal itself. Empirical mode decomposition (EMD) is widely known to be capable of eliminating the noise effects, and was introduced in the study for the de-noising. The basic functions of the EMD method are derived from the signal itself, so the method can be used to analyze nonstationary and nonlinear processes [27].

By using EMD, noisy signals can be decomposed into a number of intrinsic mode functions (imfs) and a residue $\mathrm{r}$. Each imf has only one frequency, and can reflect the intrinsic and real information of the signal [28]. Taking Test 3 as an example, the temporal waveform of the original COF signals and its power spectral density (PSD) spectra are respectively shown in the top left-hand-side and right-hand-side diagrams of Fig. 2. It can be clearly observed that the raw COF signals contained two frequency components before decomposition. Therefore, the first two decomposed imfs (imf1 and imf2) and their PSD spectra are shown in the successive left-hand-side and right-hand-side diagrams, respectively. Obviously, the waveforms of imf1 and imf2, and their PSD spectra displayed in Figs. 2(c)-2(f) show that they are nearly monocomponent, whilst the frequency of the first two imfs coincides with the dominant frequencies of the original signals, which is approximately $110.2 \mathrm{~Hz}$ and $79.2 \mathrm{~Hz}$, separately. Thus, it can be confirmed that these two imfs mainly correspond to the noise components.

The de-noised COF signals throughout friction process of four tests are shown in Fig. 3. Although the 

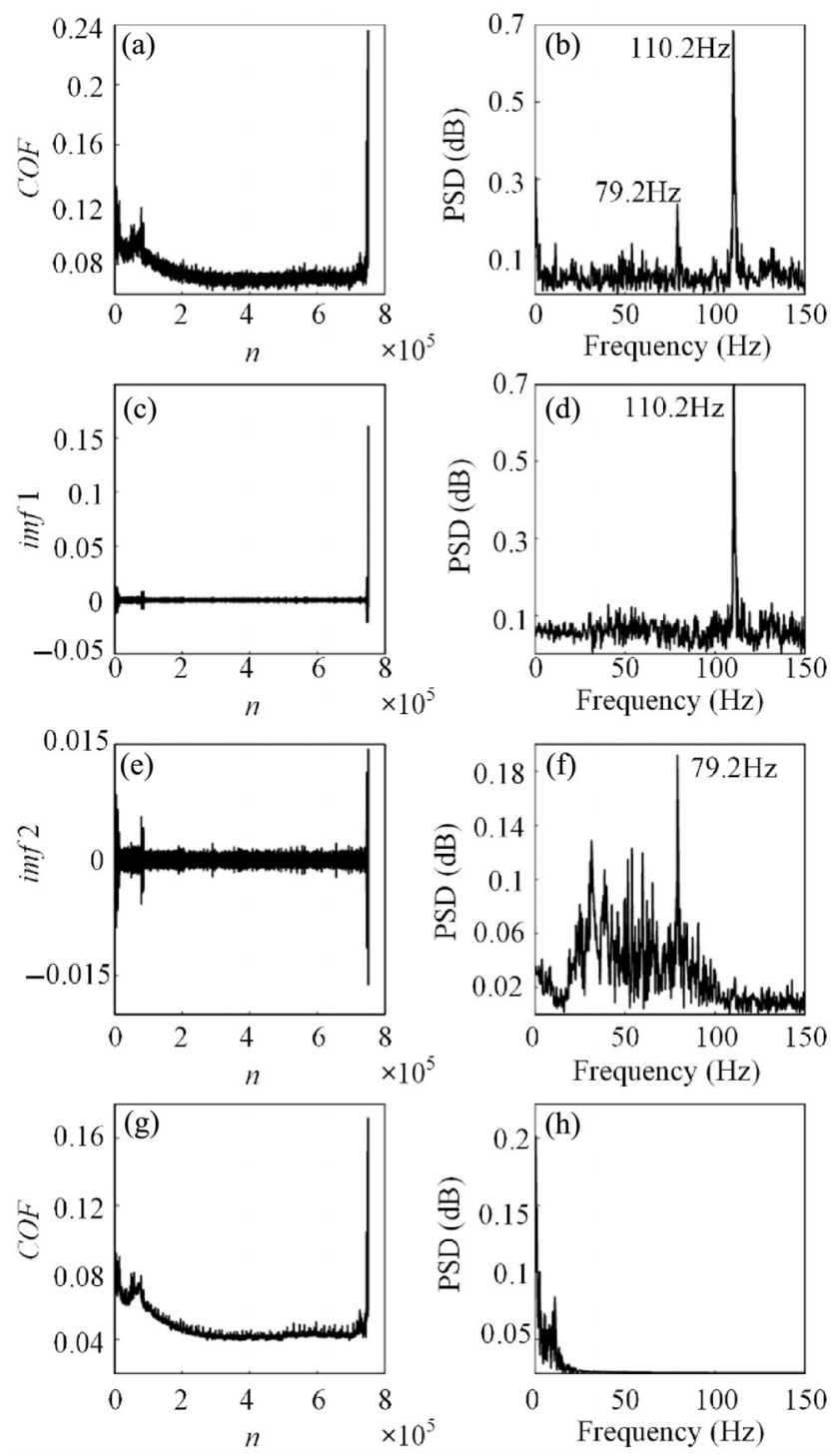

Fig. 2 Partial results of EMD decomposition and corresponding PSD spectra graph.

wear lives in four tests are not the same on account of different working conditions, the COF signals evolve in a similar way, which can be mainly divided in three stages: the running-in, steady-state, and increasing friction stages. The COF signals first increased, quickly reaching a peak value in the running-in friction process (Fig. 3(b)(i)), then gradually decreased and remained in a stable value in steady-state friction process (Fig. 3(b)(ii)), and the COF sharply increased in the increasing process (Fig. 3(b)(iii)). Additionally, there were fluctuations in signals at mean value, especially in the running-in and increasing friction stages.
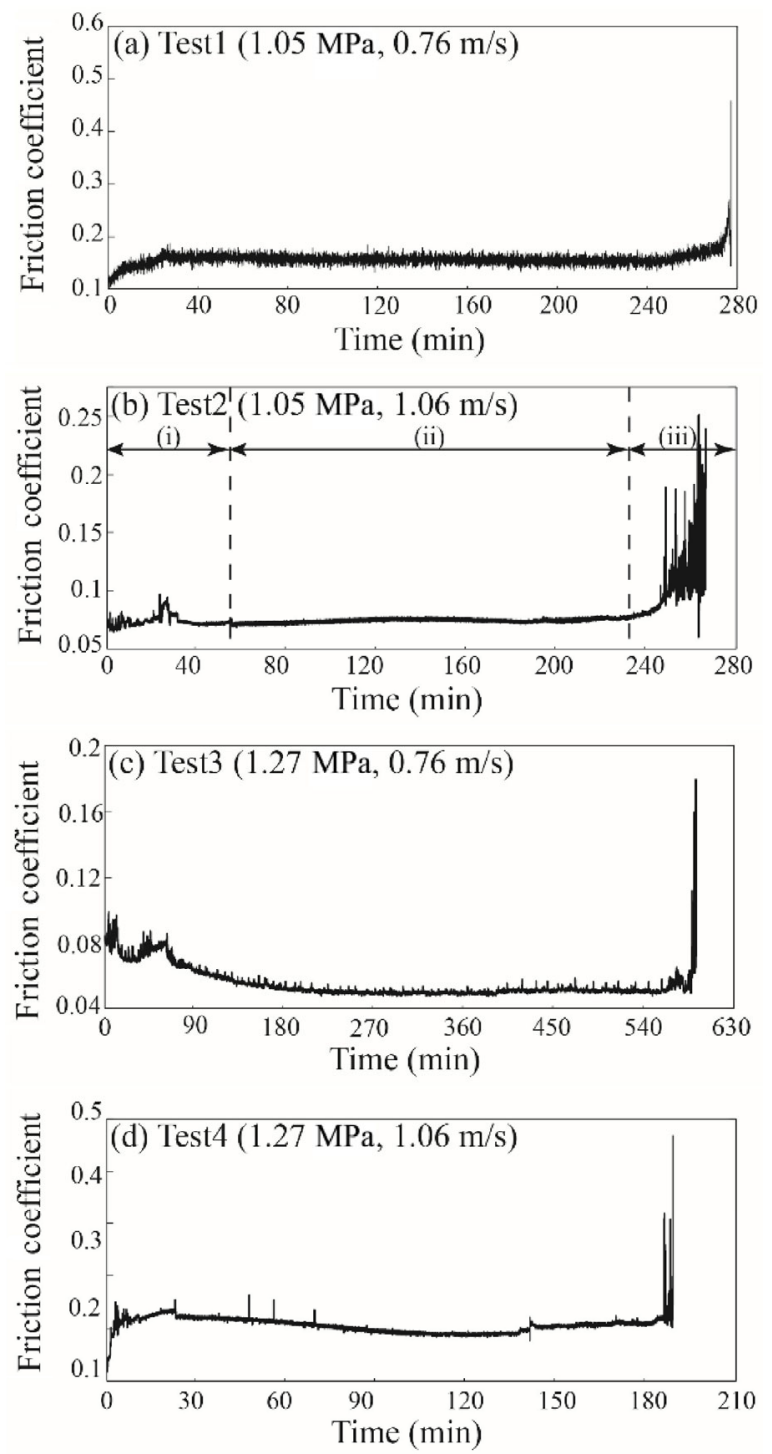

Fig. 3 COF signals during the rotating friction process.

\section{Feature parameter extraction of chaotic attractors}

\subsection{Phase space reconstruction}

Reconstructing the friction signal time series into an appropriate high dimension phase space is an effective means to investigate the chaotic characteristics of the friction process in a tribological system. According to the time delay embedding technique proposed by Takens, the method of delays can be used to embed a scalar time series $\left\{x_{i}\right\}(i=1,2,3, \ldots, N)$ into an $m$-dimension space as follows [29]: 


$$
X_{i}=\left[x_{i}, x_{i+\tau}, x_{i+2 \tau}, \ldots x_{i+(m-1) \tau}\right], i=1,2, \ldots, N
$$

The phase space can be expressed as follows:

$$
X=\left[\begin{array}{c}
X_{1} \\
X_{2} \\
\vdots \\
X_{N}
\end{array}\right]=\left[\begin{array}{cccc}
x_{1} & x_{1+\tau} & \cdots & x_{1+(m-1) \tau} \\
x_{2} & x_{2+\tau} & \cdots & x_{2+(m-1) \tau} \\
\vdots & \vdots & \ddots & \vdots \\
x_{N} & x_{N+\tau} & \cdots & x_{N+(m-1) \tau}
\end{array}\right]
$$

where $N$ is the number of the phase space vector and $N=n-(m-1) \tau, \tau$ is the delay time and $m$ is the embedding dimension.

Takens' theorem assumes that the time delay $\tau$ can be chosen almost arbitrarily in the case of an infinite noise-free data set [30]. However, the real data sets are finite and noisy; thus, the selection of embedding parameters plays a significant role in the construction of the phase space. In the presented work, $\tau$ and $m$ are established by applying the mutual information (MI) method and the false nearest neighbors (FNN) method, separately. As suggested in Refs. [31, 32], $\tau$ can be determined as the first minimum of the MI function, and according to Refs. [33, 34], the phase space is entirely opened when the percentage of the FNN point decreases to $5 \%$, and the optimal embedding dimension $m$ is computed.

\subsection{Recurrence plot and recurrence rate $R R$}

A recurrence plot $(\mathrm{RP})$, a visualization tool introduced by Eckmann et al. [35], is presented to visualize recurrences in multi-dimensional phase space. Concretely, it is a two dimensional graphical representation of the trajectory of the dynamical system in the form of a binary recurrence matrix $R$, and the entry $R_{i, j}$ is expressed as follows:

$$
R_{i, j}=\Theta\left(\varepsilon-\left\|X_{i}-X_{j}\right\|\right), X_{i}, X_{j} \in R^{m}, i, j=1,2, \ldots, N
$$

where $R_{i, j}$ is an element of the recurrence matrix; $N$ is the number of the vector $\left\{X_{i}\right\}(i=1,2,3, \ldots, N) ; \varepsilon$ is the threshold distance; the notation $\|\cdot\|$ is calculated as a Euclidean norm in the phase space, and $\Theta(\cdot)$ is the Heaviside function, defined as follows:

$$
\Theta(x)= \begin{cases}0, & x \leq 0 \\ 1, & x>0\end{cases}
$$

Owing to unnecessary prior assumptions on statistical properties, the RPs, along with RQA, are applicable to the characterization of nonlinear time series and have gained an increasing amount of attention in a variety of fields [36,37]. The recurrence for a time series is when a point on the trajectory repeats itself, which means that a point is close enough to another one within a suitably selected interval of error [38]. The basic measure of the RQA is the recurrence rate (RR) [39], which measures the percentage of darkened pixels in an RP:

$$
\mathrm{RR}=\frac{1}{N^{2}} \sum_{i, j=1}^{N} R_{i, j}
$$

$\mathrm{RR}$ is a measure of the density of recurrence points and corresponds to the correlation integral. However, the calculation of correlation dimension needs a large amount of data points, and the determination of the scaling region is relatively subjective. We can avoid the constraints with RR. By employing the definition of the $R_{i, j}$ in Eq. (4), we can directly relate RR to $\varepsilon$ :

$$
\operatorname{RR}(\varepsilon)=\frac{1}{N^{2}} \sum_{i, j=1}^{N} \Theta\left(\varepsilon-\left\|X_{i}-X_{j}\right\|\right)
$$

An RR value, ranging from 0 to 1 , is a measure based on overall probability that a certain state recurs in an RP. Given a specified $\varepsilon$, a large RR indicates an intensive distribution of phase points and a high degree of convergence. Furthermore, a trajectory with a larger RR has a stronger space-filling capacity.

\subsection{Feature parameter $R$ of chaotic attractors}

In order to extract a quantitative characterization of the size range where running-in attractors exist, the recurrence matrix and RPs are obtained based on phase space reconstruction, and the relationship between RR and $\varepsilon$ is defined as in Eq. (7) is calculated. The cutoff distance $\varepsilon$ defines a hypersphere centered at phase points, every point in the phase space is a neighbor of every other point when $\varepsilon$ is large enough, and the phase space will be included in a hypersphere of radius $\varepsilon$, which can be defined as the enclosing radius $R$. $R$ can be computed by the plot of RR and $\varepsilon$, and the eigenvalue corresponds to the first abscissa when $R R=1$. The phase points can be completely 
enclosed when $\varepsilon$ is greater than $R$.

We take the typical Lorenz chaotic attractor, which is defined by Eq. (8), as an example to illustrate the calculation of $R$. The differential equations are solved numerically by the four-order Runge-Kutta method with a step of 0.01 and iterating time is $150 \mathrm{~s}$, after discarding the initial transient points to allow the trajectory to fall to the attractor. A file containing 4000 data points is obtained as a time series.

$$
\left\{\begin{array}{l}
\dot{x}=\sigma(y-x) \\
\dot{y}=r x-x z-y \\
\dot{z}=x y-b z
\end{array}\right.
$$

where, $\sigma=10, r=28, b=8 / 3$. The initial values of $x, y$, and $z$ are 10,20 , and -10 , respectively.

The determination procedure of embedding parameters of the Lorenze time-series (Fig. 4(a)) are shown in Figs. 4 (b) $-4(\mathrm{c})$, $\tau$ corresponds to the first minimum of the MI function, and the percentage of FNN stabilizes after $m=4$, as indicated by the filled dot. Therefore, for the given time series, $\tau=15$ and $m=4$ are used for the phase space reconstruction and the following analysis. The RPs of the Lorenz time series dependent on the cutoff distance $\varepsilon$ are shown in Fig. 5.

As shown in Fig. 5, a fixed $\varepsilon$ results in a symmetric $\mathrm{RP}$, and the RPs exhibit characteristic small-scale patterns that are caused by different $\varepsilon$ values. When $\varepsilon$ is relatively small, there are few recurrence points,
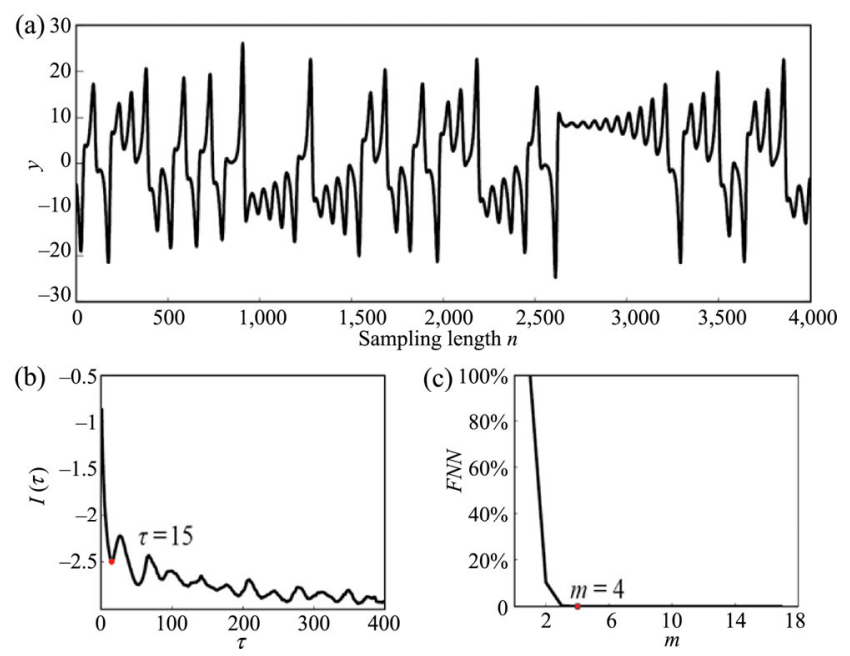

Fig. 4 One-dimensional time series $y$ of the Lorenz system and embedding parameters. and the RP is sparse, with an increase in $\varepsilon$, the pairs of recurrence point increase, and the black area is larger than the white area within an RP. Figure 6 illustrates how the $\varepsilon$ can determine RR. RR was calculated by a fixed $\varepsilon$ and using a Euclidean distance. From results shown in Fig. 6, RR first increases and then tends to stabilize with increasing $\varepsilon$, and $R$ of the given theoretical time series is 61.72. It is important to note that the magnitude of the parameter is not related to whether the attractor is chaotic, but is a quantitative representation of the volume and degree of convergence of the attractors. A lower magnitude of $R$ indicates that in a smaller volume where the attractors exist and a higher degree of convergence of phase points. (a)

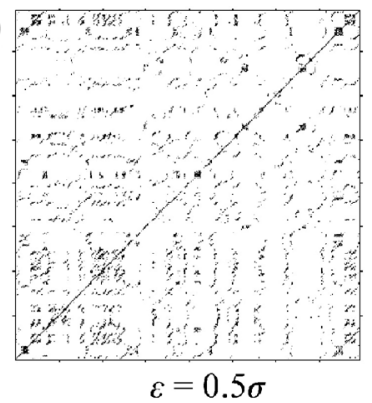

(c)

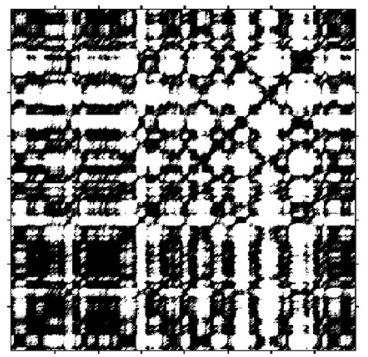

$\varepsilon=1.5 \sigma$ (b)

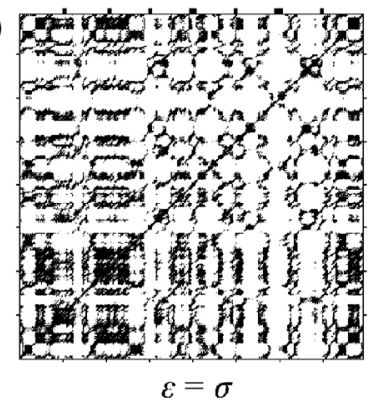

(d)

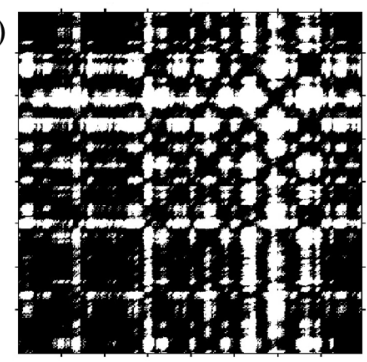

$\varepsilon=2 \sigma$
Fig. 5 RPs of the Lorenz time series dependent on cutoff distance $\varepsilon ; \sigma$ is the variance of the given series.

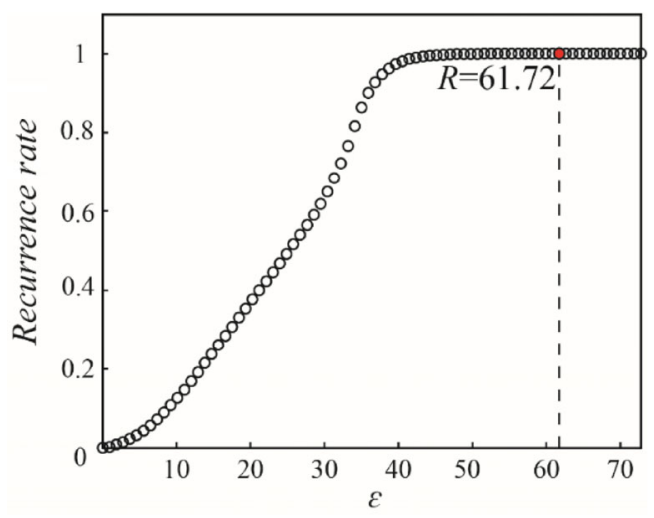

Fig. 6 Sketch map of computing enclosing radius $R$ based on RR. 


\section{Boundedness of running-in attractors}

\subsection{Recurrence plots of COF signals}

As noted above, such recurrence of a state at time $i$ at a different time $j$ is pictured within an RP with black and white dots, where black dots mark a recurrence. It has been generally confirmed that the visual inspection of RPs reveals four typical large-scale patterns (the typology): homogeneous, periodic, drift, and disrupted [40]. The typology of the RPs can qualitatively give hints about specific dynamic behaviors of a system. The first two patterns correspond to stationary systems with short relaxation times and periodic (or quasi-periodic) systems, respectively. On the contrary, non-stationary systems with slowly varying parameters have RPs paled away from the LOI (line of identity with an angle of $\pi / 4$ ) and can be classified as drift, while abrupt changes or extreme events in dynamics cause white areas and lead to a disrupted RP.
The embedding parameters of the four tests were established by applying the method introduced in Section 3, and the results are summarized in Table 2. The recurrence matrices of the COF signals were calculated by applying a threshold such that RR was $50 \%$ and using the Euclidean norm, the RPs are then obtained via the MATLAB CRP Toolbox [41]. For shorter computational time and smaller memory requirements, the COF signals were divided into non-overlapping subsequences with an equal length of 23785 data points, and only the initial 3785 points were calculated at every subsequence. Owing to space limitations, this paper enumerates only the 16 profiles in Test 2, as displayed in Fig. 7.

Table 2 Optimal time delay and embedding dimension of friction coefficient in friction process.

\begin{tabular}{ccccc}
\hline & Test 1 & Test 2 & Test 3 & Test 4 \\
\hline Time delay $\tau$ & 22 & 21 & 17 & 25 \\
$\begin{array}{c}\text { Embedding } \\
\text { dimension } m\end{array}$ & 7 & 15 & 19 & 13 \\
\hline
\end{tabular}

(a)

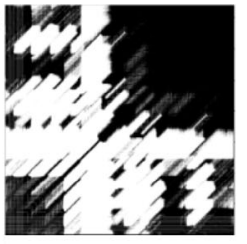

$0-17 \mathrm{~min}$

(e)

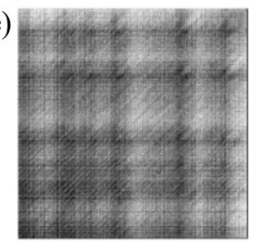

$68-85 \mathrm{~min}$

(i)

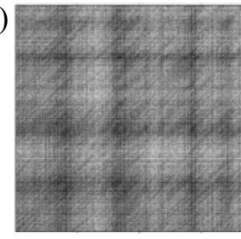

$136-153 \mathrm{~min}$

(m)

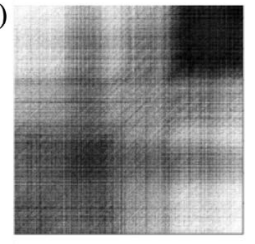

204-221 min

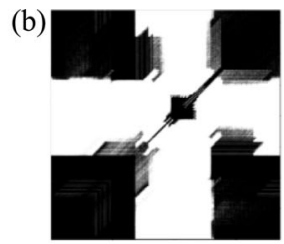

17-34 min

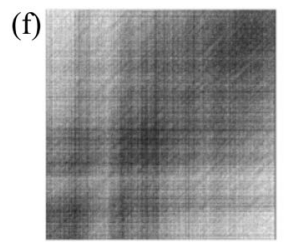

85-102 min

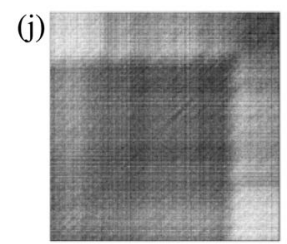

$153-170 \mathrm{~min}$

(n)

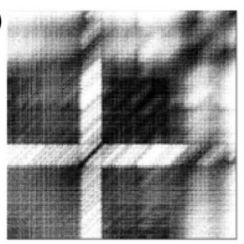

$221-238 \mathrm{~min}$

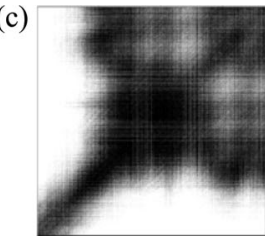

$34-51 \mathrm{~min}$

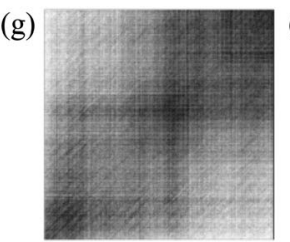

102-119 min

(k)

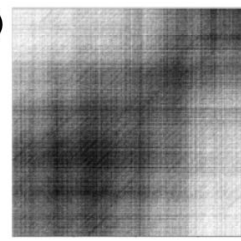

$170-187 \mathrm{~min}$

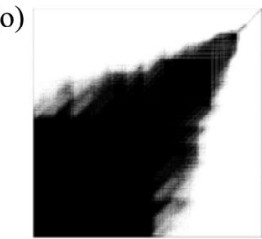

238-255 min

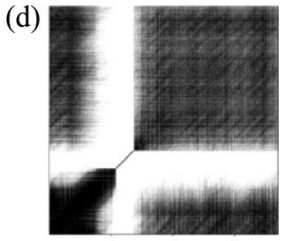

51-68 min

(h)

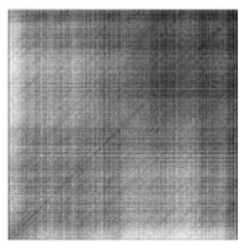

119-136 min

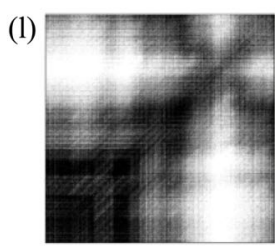

187-204 min

(p)

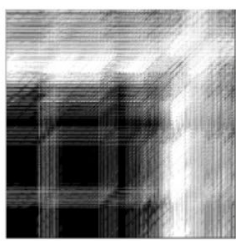

255-272 min

Fig. 7 Evolvement of RPs of the $C O F$ signal of Test 2 during the friction process. 
An overview of the RPs' evolvement in the running-in attractors throughout the friction process is illustrated in Fig. 7, and the macroscopic patterns of RPs depict an evolution of "disrupted-drifteddisrupted". It can be observed in Figs. 7(a)-7(d) that large black and white areas occur at the corner of the RPs, indicating the friction system is unstable during the period. The disrupted typology corresponds to a violent fluctuation and a sparse distribution of phase points. In Figs. $7(\mathrm{e})-7(\mathrm{~m})$, there is a fine structure of vertical (horizontal) lines, essentially demonstrating that the phase space trajectory does not change or changes very slowly. It can be recognized as typical behavior of laminar states [42]. Interestingly, there is a checkerboard structure in Fig. 7(n); it seems plausible that on this time-scale the underlying states strongly deviate from the previous "laminar" states. After wearing for $238 \mathrm{~min}$, the COF signal changes immensely and a large fluctuation starts to appear. The RPs shown in Figs. 7(o)-7(p) are characterized with large black regions concentrated at the lower left corner and can be regarded as disrupted patterns.

The RPs evolve from a disrupted pattern to a drifted pattern during the running-in process, maintain the drifted pattern in the steady-state process, and finally return to a disrupted pattern in the increasing friction process. Although the amplitude analysis of the friction coefficient was more likely not accurately revealed, the qualitative features can be extracted for comparison.

\subsection{Feature parameters of running-in attractors}

In order to illustrate the applicability of the proposed running-in attractors quantitative measurements of $\mathrm{COF}, R$ and $\mathrm{RR}$ are computed for each signal, and the evolution of $R$ and RR are displayed in Fig. 8.

As shown in Fig. 8, in different friction stages, the characteristic parameters $R$ and RR show temporal variation and preferable regularity. Within the running-in friction process, $R$ shows a downward trend; during the steady-state friction period, $R$ becomes smooth and trends towards the small steady values; and $R$ shows an upward trend until failure occurs. On the contrary, RR increases at first, then maintains stability, and finally increases. It is shown that both the $R$ and RR values computed from different tests evolve in a similar way, and the evolution is synchronous if both eigenvalues are extracted from one friction coefficient signal. The results of $R$ and
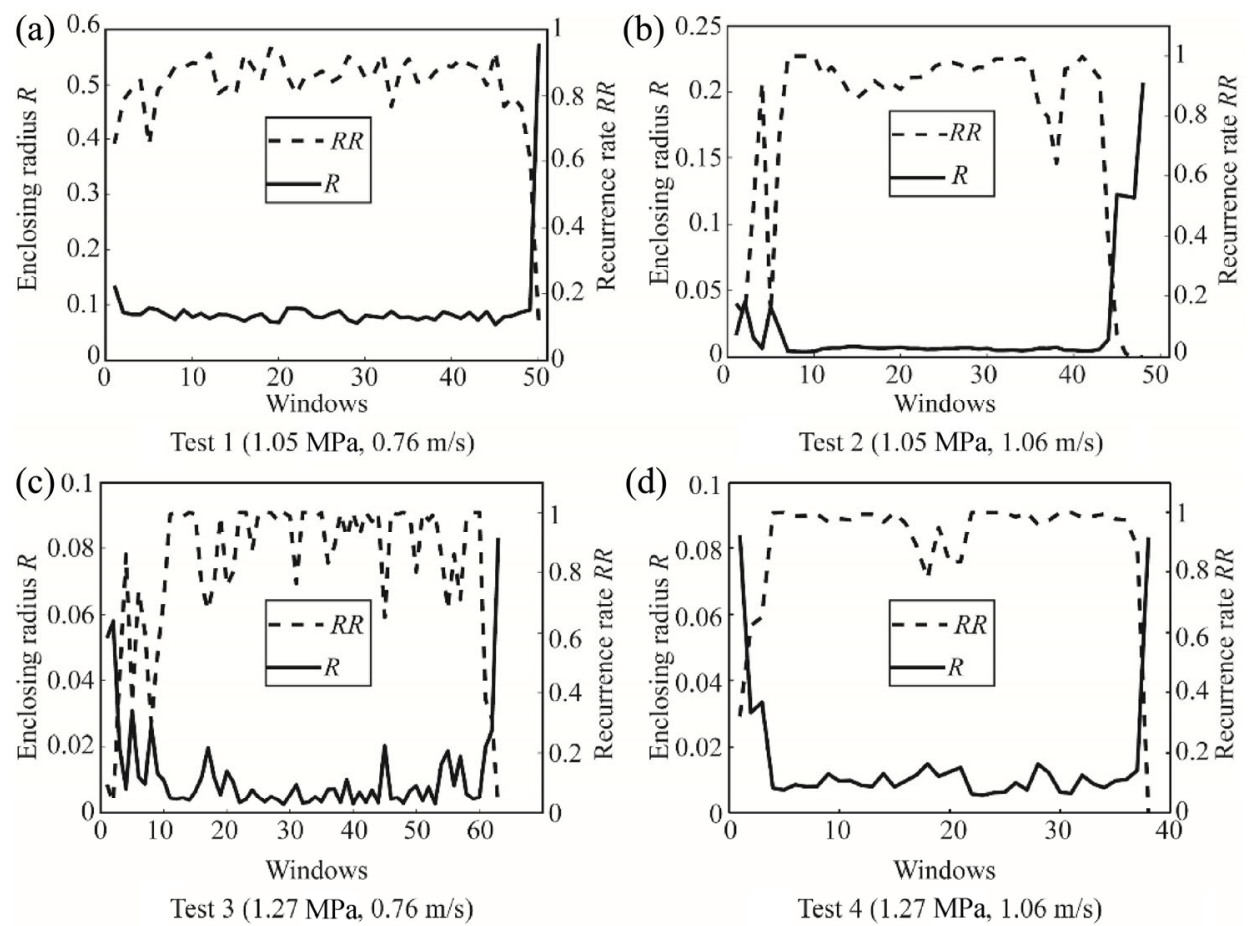

Fig. 8 Evolvement of $R$ and RR of running-in attractors during the friction process. 
RR correspond with the typology of RPs evolution displayed in Fig. 7.

The Pearson correlation $r$ is adapted as the statistic for measuring the association of the changes in two parameters. As shown in Table 3, the Pearson correlation $r$ of $R$ and RR obtained by the four tests are negative, and the absolute values are all greater than 0.76 , indicating there is a fine negative correlation between the two parameters.

$$
r=\frac{\operatorname{cov}(R, \mathrm{RR})}{\sqrt{\operatorname{var}(R) \cdot \operatorname{var}(\mathrm{RR})}}
$$

\subsection{Discussion}

The phase trajectory is an effective tool for describing the time evolution process of a system intuitively; thus, it is perfectly suitable for analyzing tribological issues. According to the evolution of the phase trajectory of friction signals, the friction process can be interpreted as "self-organization, chaos, and system-instability", which correspond to the patterns of "running-in, steady-state, and increasing" across the friction process.

Owing to the rather rough initial machined surfaces, the contact pressure was large and the friction was severe at the very beginning of the experiments. The COF significantly fluctuated, indicating a sparse distribution of phase points and a high degree of divergence. The typology of RPs could be interpreted as disrupted, and the bounds of friction coefficient attractors were rather large. $R$ was rather large, while RR was small.

Within the running-in friction period, asperities grew increasingly abundant and came into contact; the friction coefficient grew stable with decreasing real contact pressure. The distribution of phase points becomes relatively concentrated, and the bound of the phase trajectory gradually converged. Hence, the running-in process can be regarded as the formation process of running-in attractors. This results in a uniformly distributed RP, a decrease in the shrinking of $R$, and an increase in RR.

In the steady-state friction stage, the friction system was well lubricated, and chiefly characterized by steady surface contact with low and stable heat generation.
Table 3 Correlation coefficient of $R$ and RR during the friction process.

\begin{tabular}{ccccc}
\hline & Test 1 & Test 2 & Test 3 & Test 4 \\
\hline $\begin{array}{c}\text { Pearson } \\
\text { correlation } r\end{array}$ & -0.8762 & -0.7636 & -0.8971 & -0.9611 \\
\hline
\end{tabular}

The friction coefficient maintained stable values, and the phase space trajectory seemed trapped during this stage. The main structures in RPs are vertical and horizontal lines, where the trajectories hover in a confined space and remain stable. Both $R$ and $R R$ were relatively stable in this stage.

When wear failure occurred, the COF increased and fluctuated sharply as a consequence of the generation of wear particles, as well as the deterioration of lubricant and worn surfaces. The phase trajectories gradually diverged and finally escaped the limited space in the increasing friction process, causing a disrupted RP. Moreover, $R$ increased and RR decreased dramatically.

Furthermore, for the same pressure, the amplitude and the fluctuation of $R$ both decreased with increasing velocity. Meanwhile, for the same velocity, the amplitude of $R$ decreased while the fluctuation increased with an increase in normal pressure. In the process of sliding friction, the actual contact and interaction of surface asperities can be dramatically reduced by the increasing velocity, leading to a stable friction coefficient. With increasing pressure, the actual contact surface and the number of interacted asperities increase, causing a smaller friction coefficient amplitude. At the same time, it can be stated that a higher pressure will result in an increasing mechanical engagement between the asperities, as well as a fluctuation in the friction coefficient.

\section{Conclusions}

The friction experiments were performed by sliding a ring (AISI 52100) against a stationary disc (AISI 1045) under lubricated conditions. The evolution in running-in attractors was investigated by defining a feature parameter $R$ extracted by RP and RQA. The dynamic evolution of friction systems was intuitively analyzed by RPs and characteristic parameters $R$ and RR. The major conclusions can be summarized as follows: 
(1) The enclosing radius $R$, defined by RP and RQA, is adapted to describe the boundedness of running-in attractors. It is an effective way to reflect the behavior of COF during friction process. A small value of $R$ indicates an intensive distribution of phase points and a high degree of convergence, while the convergence of trajectory indicates a decrease of energy dissipation.

(2) $R$ goes through a rapid downward path during the running-in period, a stable fluctuation in the steady-state friction stage, and an uptrend in the increasing friction stage, following the same trend as the COF in time domain. The identification of a friction state with $R$ is consistent with that using the COF signals; therefore, $R$ can be used to monitor the friction state of tribopairs.

(3) The results of RPs and RQA indicate that the non-linear dynamic behavior of the friction process can be described by the pattern of RPs and RQA measures. The drifted typology of RPs, the decrease in $R$, and the increase in RR occur when the running-in process is terminated. Additionally, the disrupted typology of RPs, the increase in $R$, and the decrease in $R R$ indicate the failure occurs.

(4) The typology of RPs and chaotic parameters of COF signals during different friction tests evolve in a similar way, which can be used for wear condition identification. Furthermore, they synchronously evolve when the signal is extracted from a friction system.

\section{Acknowledgments}

This work is carried out within the projects supported by the National Natural Science Foundation of China (Nos. 51775546 and 51375480) and the Priority Academic Program Development of Jiangsu Higher Education Institutions.

Open Access: The articles published in this journal are distributed under the terms of the Creative Commons Attribution 4.0 International License (http:// creativecommons.org/licenses/by/4.0/), which permits unrestricted use, distribution, and reproduction in any medium, provided you give appropriate credit to the original author(s) and the source, provide a link to the Creative Commons license, and indicate if changes were made.

\section{References}

[1] Blau P J. The significance and use of the friction coefficient. Tribol Int 34(9): 585-591 (2001)

[2] Blau P J. A model for run-in and other transitions in sliding friction. J Tribol 109(3): 537-543 (1987)

[3] Xie Y B. Theory of Tribo-systems. In Tribology-Lubricants and Lubrication. Kuo C H, Ed. InTech, 2011.

[4] Urbakh M, Klafter J, Gourdon D, Israelachvili J. The nonlinear nature of friction. Nature 430(6999): 525-528 (2004)

[5] Xia X T, Chen L, Fu L L, Li J H. Information mining for friction torque of rolling bearing for space applications using chaotic theory. Res J Appl Sci Eng Technol 5(22): 5223-5229 (2013)

[6] Vitanov N K, Hoffmann N P, Wernitz B. Nonlinear time series analysis of vibration data from a friction brake: SSA, PCA, and MFDFA. Chaos Solit Fract 69: 90-99 (2014)

[7] Enns R H, McGuire G C. Nonlinear Physics with Mathematica for Scientists and Engineers. Boston: Birkhäuser, 2001.

[8] Zhou Y K, Zhu H, Zuo X. Dynamic evolutionary consistency between friction force and friction temperature from the perspective of morphology and structure of phase trajectory. Tribol Int 94: 606-615 (2016)

[9] Zhu H, Ge S R, Li G, Lv L. Test of running-in process and preliminary analysis of running-in attractors. Lubr Eng 32(1): 1-3 (2017)

[10] Grassberger P, Procaccia I. Characterization of strange attractors. Phys Rev Lett 50(5): 346-349 (1983)

[11] Lopes R, Betrouni N. Fractal and multifractal analysis: a review. Med Image Anal 13(4): 634-649 (2009)

[12] Wolf A, Swift J B, Swinney H L, Vastano J A. Determining Lyapunov exponents from a time series. Phys D: Nonlinear Phenomena 16(3): 285-317 (1985)

[13] Fraser A M. Information and entropy in strange attractors. IEEE Trans Inf Theory 35(2): 245-262 (1989)

[14] Manuca R, Savit R. Stationarity and nonstationarity in time series analysis. Phys D: Nonlinear Phenomena 99(2-3): 134-161 (1996)

[15] Zhou Y K, Zhu H, Zuo X. The behavior of intrinsic randomness and dynamic abrupt changes of friction force signal during the friction process. $J$ Tribol 138(3): 031605 (2016)

[16] Zhu H, Ge S R, Lv L, Lu B B. Evolvement rule of running-in attractor. Chin J Mech Eng 44(3): 99-104 (2008)

[17] Sun D, Li G B, Wei H J, Liao H F. Experimental study on the chaotic attractor evolvement of the friction vibration in a running-in process. Tribol Int 88: 290-297 (2015) 
[18] Zhou Y K, Zhu H, Zuo X, Yang J H. Chaotic characteristics of measured temperatures during sliding friction. Wear 317(1-2): 17-25 (2014)

[19] Liu T, Li G B, Wei H J, Sun D. Experimental observation of cross correlation between tangential friction vibration and normal friction vibration in a running-in process. Tribol Int 97: 77-88 (2016)

[20] Jiang Y, Zhu H, Li Z, Peng Z. The nonlinear dynamics response of cracked gear system in a coal cutter taking environmental multi-frequency excitation forces into consideration. Nonlinear Dyn 84(1): 203-222 (2016)

[21] Manevitch L I, Kovaleva A S, Manevitch E L, Shepelev D S. Limiting phase trajectories and non-stationary resonance oscillations of the Duffing oscillator. Part 1. A non-dissipative oscillator. Commun Nonlinear Sci Numer Simul 16(2): 1089-1097 (2011)

[22] Manevitch L I, Kovaleva A S, Manevitch E L, Shepelev D S. Limiting phase trajectories and nonstationary resonance oscillations of the Duffing oscillator. Part 2. A dissipative oscillator. Commun Nonlinear Sci Numer Simul 16(2): 1098-1105 (2011)

[23] Zhang F C, Shu Y L, Yang H L. Bounds for a new chaotic system and its application in chaos synchronization. Commun Nonlinear Sci Numer Simul 16(3): 1501-1508 (2011)

[24] Gao J B. Detecting nonstationarity and state transitions in a time series. Phys Rev E 63(6): 066202 (2001)

[25] Marwan N, Wessel N, Meyerfeldt U, Schirdewan A, Kurths J. Recurrence-plot-based measures of complexity and their application to heart-rate-variability data. Phys Rev E 66(2): 026702 (2002)

[26] Jackson R L, Green I. Study of the tribological behavior of a thrust washer bearing. Tribol Trans 44(3): 504-508 (2001)

[27] Boudraa A O, Cexus J C. EMD-Based signal filtering. IEEE Trans Instrum Meas 56(6): 2196-2202 (2007)

[28] Peng Z K, Tse P W, Chu F L. An improved Hilbert-Huang transform and its application in vibration signal analysis. $J$ Sound Vibr 286(1-2): 187-205 (2005)

[29] Takens F. Detecting strange attractors in turbulence. In Dynamical Systems and Turbulence, Warwick 1980. Lecture Notes in Mathematics, vol 898. Rand D, Young L S, Eds. Berlin, Heidelberg: Springer. 1981: 366-381.
[30] Kim H S, Eykholt R, Salas J D. Nonlinear dynamics, delay times, and embedding windows. Phys D: Nonlinear Phenomena 127(1-2): 48-60 (1999)

[31] Piórek M. Mutual information for quaternion time series. In Computer Information Systems and Industrial Management. CISIM 2016. Lecture Notes in Computer Science, vol 9842. Saeed K, Homenda W, Eds. Cham: Springer, 2016: 453-461.

[32] Fraser A M, Swinney H L. Independent coordinates for strange attractors from mutual information. Phys Rev A 33(2): 1134-1140 (1986)

[33] Kennel M B, Brown R, Abarbanel H D I. Determining embedding dimension for phase-space reconstruction using a geometrical construction. Phys Rev A 45(6): 3403 (1992)

[34] Liu L J, Fu Y, Ma S W. Wind power short-term prediction method based on multivariable mutual information and phase space reconstruction. In Intelligent Computing in Smart Grid and Electrical Vehicles. ICSEE 2014, LSMS 2014. Communications in Computer and Information Science, vol 463. Li K, Xue Y, Cui S, Niu Q, Eds. Berlin, Heidelberg: Springer, 2014: 1-12.

[35] Eckmann J P, Kamphorst S O, Ruelle D. Recurrence plots of dynamical systems. EPL (Europhys Lett) 4(9): 973-977 (1987)

[36] Marwan N, Kurths J, Saparin P. Generalised recurrence plot analysis for spatial data. Phys Lett A 360(4-5): 545-551 (2007)

[37] Addo P M, Billio M, Guégan D. Nonlinear dynamics and recurrence plots for detecting financial crisis. North Amer $J$ Econ Finance 26: 416-435 (2013)

[38] Llop M F, Gascons N, Llauró F X. Recurrence plots to characterize gas-solid fluidization regimes. Int $J$ Multiph Flow 73: 43-56 (2015)

[39] Oberst S, Lai J C S. Chaos in brake squeal noise. J Sound Vibr 330(5): 955-975 (2011)

[40] Marwan N, Romano M C, Thiel M, Kurths J. Recurrence plots for the analysis of complex systems. Phys Rep 438(5-6): 237-329 (2007)

[41] Marwan N, Kurths J, Foerster S. Analysing spatially extended high-dimensional dynamics by recurrence plots. Phys Lett A 379(10-11): 894-900 (2015)

[42] Marwan N, Kurths J. Line structures in recurrence plots. Phys Lett A 336(4-5): 349-357 (2005) 


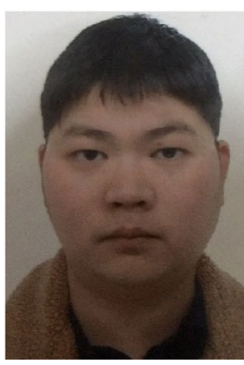

Guodong SUN. He received his bachelor degree in mechanical engineering in 2013 from Nanjing University of Science and Technology, Nanjing, China. After then, he is a

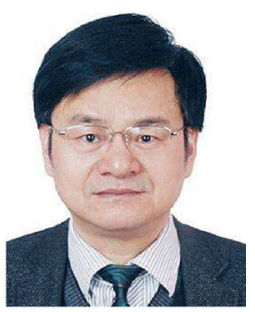

Hua ZHU. He received his Ph.D degree in School of Mechatronic Engineering from China University of Mining and Technology, Xuzhou, China. His current position is a
Ph.D student in the School of Mechatronic Engineering of China University of Mining and Technology, Xuzhou, China. His research interests include friction signals and chaos theory.

professor, the supervisor of Ph.D. students and the deputy director of the Mine Robot Research Institute. His research areas cover the tribology theory and application, fractal and chaos theory, noise and vibration control, and mine rescue robot research. 